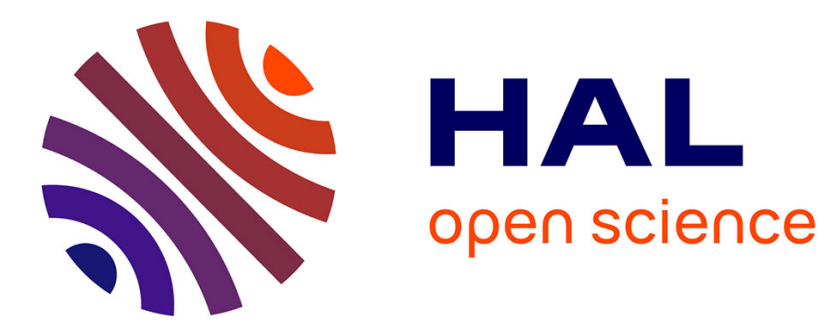

\title{
La construction du sujet souffrant au travail au travers des instruments scientifiques de mesure
}

\author{
Florence Allard-Poesi, Sandrine Hollet-Haudebert
}

\section{To cite this version:}

Florence Allard-Poesi, Sandrine Hollet-Haudebert. La construction du sujet souffrant au travail au travers des instruments scientifiques de mesure. @GRH, 2012, 2012/4 (5), pp.45-74. 10.3917/grh.124.0045 . hal-01251213

\section{HAL Id: hal-01251213 \\ https://hal.science/hal-01251213}

Submitted on 8 Jan 2016

HAL is a multi-disciplinary open access archive for the deposit and dissemination of scientific research documents, whether they are published or not. The documents may come from teaching and research institutions in France or abroad, or from public or private research centers.
L'archive ouverte pluridisciplinaire HAL, est destinée au dépôt et à la diffusion de documents scientifiques de niveau recherche, publiés ou non, émanant des établissements d'enseignement et de recherche français ou étrangers, des laboratoires publics ou privés. 


\title{
La construction du sujet souffrant au travail au travers des
}

\author{
instruments scientifiques de mesure
}

Florence Allard-Poesi -auteur correspondant-

allard-poesi@u-pec.fr

\author{
Sandrine Hollet-Haudebert \\ $\underline{\text { hollet@u-pec.fr }}$
}

Université Paris-Est

Institut de Recherche en Gestion

$@ G R H, 2012 / 4, \mathbf{n}^{\circ} 5$ pages 45 à 74

\section{Résumé :}

S'inscrivant dans une perspective foucaldienne, cette recherche a pour objectif de cerner les traits et caractéristiques essentiels du sujet que construisent les méthodes scientifiques d'évaluation de la souffrance au travail. Empruntant une démarche qualitative, nous analysons en profondeur sept échelles d'auto-évaluation de la souffrance au travail couramment utilisées en management. Notre recherche montre que $1 /$ ces échelles véhiculent, à l'instar d'autres techniques de mesure utilisées en gestion des ressources humaines (cf. Townley, 1993 ; Pezet, 2007), les idées d'un individu calculable et comptable de lui-même. Elle précise ce second aspect en montrant que les échelles d'évaluation de souffrance construisent un individu 2/ capable d'enregistrer et d'évaluer les évènements qui l'affectent et 3/ qui entretient des relations instrumentales avec le monde et les autres. Notre recherche montre enfin que 4/ la souffrance ne paraît pas tant importer pour elle-même qu'en tant qu'elle est susceptible de réduire l'activité du sujet souffrant. Les échelles d'évaluation de la souffrance fabriquent ainsi un sujet qui, s'il est rationnel, n'a aucune prise sur son environnement de travail. 
En s’intéressant à la manière dont les échelles de mesure voient le sujet souffrant, notre recherche complète les travaux s'inscrivant dans une perspective critique de la santé au travail qui ont principalement cherché à mettre en lumière les points aveugles des recherches orthodoxes. Nos résultats précisent ainsi les traits de l'individu 'comptable' mis en évidence par les recherches antérieures et permettent de contraster ces traits d'avec ceux de l'individu libre, 'entrepreneur de lui-même' véhiculé par les discours managériaux (Rose, 1999). Les conséquences théoriques, méthodologiques et pratiques de ces résultats sont envisagées.

Mots clés : Echelle de mesure, Foucault, Savoir, Souffrance au travail, Sujet.

\section{How Scientific Measures of Psychological Distress at Work Construct the Suffering Subject}

Following a Foucaldian perspective, this research aims to decipher the traits and characteristics of the subject that are constructed by the scientific scales designed to measure psychological distress at work. We rely on a qualitative approach and analyze seven selfreported measures of psychological distress widely used in management science. The analysis shows that $1 /$ these measures, along with other measures used in human resources management (Townley, 1993; Pezet, 2007), convey the idea of a calculable and accountable subject. Our results show that 'calculability' here means that 1 / the individual is able to record and evaluate the events that affect him and $2 /$ his/her relationships with others and the world are, for the most part, instrumental. Finally, our research demonstrates that psychological distress is not important in it-self but only in so far as it may reduce the activity level of the suffering subject. The scientific measures of psychological distress construct a subject that, while rational, has no influence on his work environment. 
Our research that investigates the way scientific measures see the suffering subject complement works which, adopting a critical perspective on health, have researched the 'blind spots' in orthodox studies. It details the traits of the accountable subject underlined by previous studies. These traits are to be contrasted with those of the free self-entrepreneur subject constructed by managerial discourses (Rose, 1999). We discuss the methodological, theoretical and managerial contributions of the research.

Key words: Foucault; scales; psychological distress at work; knowledge; subject.

\section{Introduction}

Depuis la fin des années 80 , le management a fait l'objet d'un nombre croissant de recherches critiques. Sous cette bannière, sont rassemblés un ensemble de travaux qui, s'appuyant sur des perspectives théoriques variées, soulignent le caractère socialement et historiquement construit tant des pratiques, des discours et des techniques de management que des connaissances élaborées sur ceux-ci et relèvent, derrière leur apparente neutralité, leur participation aux mécanismes de pouvoir et phénomènes de domination à l'œuvre dans les organisations (voir Golsorkhi, Huault, \& Leca, 2009 pour une contribution récente dans le champ de la recherche francophone). Dans cette perspective critique, les effets des méthodes de management en place, qu'il s'agisse de techniques (les méthodes de sélection ou d'évaluation des personnels par exemple, Townley, 1993 ; 1995), de modes d'organisation ou d'animation (le recours généralisé aux méthodes de contrôle de gestion, Ezzamel, Willmott, \& Worthington, 2008 ; le 'Team Management', Knights \& McCabe, 2003) font l'objet d'une attention particulière : normalisation des comportements et assujettissement des personnes à la discipline managériale pour les premiers travaux (Knights \& Willmott, 1989 ; McKinlay \& 
Starkey, 1998), sentiment d'insécurité, peurs et souffrance au travail plus récemment (Perret, 2009).

Revendiquant une approche critique de la santé au travail, certains travaux en sciences sociales (en sociologie notamment) voire interdisciplinaires (Duncan, 2007 ; Daykin \& Doyal, 1999), se sont intéressés à la manière dont les recherches scientifiques étudient et construisent leurs objets : les 'risques' (Fox, 1999), les 'maladies professionnelles' (Watterson, 1999), les ‘accidents' (Nichols, 1999). Quoique peu nombreuses, quelques recherches soulignent que la santé mentale en général, et le stress en particulier (voir Payne, 1999) sont définis et envisagés de manière trop restreinte dans les enquêtes épidémiologiques. Rabattant souvent le travail à l'emploi rémunéré en effet, ces enquêtes occultent le contexte sociopolitique plus large au sein duquel le travail s'exerce et négligent, dans le cas des femmes par exemple, la complexité et la diversité des facteurs à l'œuvre dans le stress ressenti.

La notion même de souffrance au travail et ses instrumentations n'ont cependant été que peu questionnées. Une attention particulière est certes portée à la souffrance psychique, mentale ou psychologique ${ }^{1}$ au travail, par une variété de paradigmes et disciplines ${ }^{2}$. Malgré tout, cette

\footnotetext{
${ }^{1}$ Les trois qualificatifs sont envisagés comme étant interchangeables dans le cadre de cet article. Par la suite, nous parlons de 'souffrance' ou de 'souffrance au travail' pour désigner cette souffrance psychologique. Au travers de ces trois qualificatifs, sont désignés un ensemble historiquement construit de phénomènes (Lhuilier, 2010) comprenant la fatigue nerveuse, le stress, la dépression ou encore l'épuisement professionnel -ensemble aujourd'hui réuni sous le terme de 'risques psychosociaux'. Ces différentes dénominations témoignent d'une évolution de la manière dont la souffrance au travail et le travail lui-même ont été appréhendés par les sciences sociales et la médecine au cours du temps. Ces différentes conceptions se rejoignent cependant dans une définition de la souffrance psychique au travail comme étant une expérience sensorielle et émotionnelle désagréable résultant d'une interaction entre l'individu, son activité professionnelle et l'environnement dans
} 
souffrance est 'là' (ou 'pas là') et ce, indépendamment des perspectives théoriques et des outils de mesure que nous utilisons pour l'appréhender; comme si on pouvait prétendre en rendre compte de manière si ce n'est objective, à tout le moins neutre.

Suivant là les recherches critiques précédemment évoquées, on peut cependant remarquer que ce n'est que récemment que la souffrance au travail est devenue objet de connaissance ; un objet en outre essentiellement conçu au travers de deux dimensions disjointes : un 'corps souffrant' d'abord, 'une psyché' en souffrance ensuite. L'adoption d'une perspective critique amène à questionner les effets de cette connaissance.

À ce titre, les recherches s'appuyant sur les travaux de Michel Foucault (voir Pezet, 2007 ; Townley, 1993 ; 1995 dans le champ de la gestion des ressources humaines), ont mis en évidence l'incidence des savoirs élaborés sur les personnes et les techniques utilisées en management, sur la conception même de l'individu dans les organisations. Les techniques et discours de savoir, ainsi, ne sont pas neutres mais fabriquent et véhiculent une certaine conception du 'sujet' : un sujet 'gouvernable' (Miller \& O'Leary, 1987), 'calculable' (Townley, 1993), ‘comptable' (Pezet, 2007).

C'est à cette question que nous souhaitons contribuer dans le cadre de cette recherche : quel est le sujet que construisent les méthodes scientifiques d'investigation de la souffrance au travail ? Quels en sont les traits caractéristiques ? Pour répondre à cette question, nous analysons en profondeur sept échelles d'auto-évaluation de la souffrance couramment utilisées en management.

\footnotetext{
lequel cette activité est réalisée. C'est cette définition que nous retenons dans le cadre de ce travail.

${ }^{2}$ Voir Cooper, Quick, \& Schabracq, 2009 pour un panorama récent de la recherche anglo-saxonne. Voir Dejours (2008), Clot (2010), et De Gaulejac (2011) pour des recherches significatives en France.
} 
Notre propos est organisé comme suit. Nous présentons dans un premier temps les grandes lignes de la perspective foucaldienne du savoir et du sujet sur laquelle cette recherche s'appuie et justifions dans ce cadre l'intérêt d'une étude des échelles d'auto-évaluation de la souffrance au travail. Les échelles étudiées dans le cadre de cette recherche et la méthode d'analyse empruntée sont ensuite exposées. Nous présentons enfin les résultats de l'analyse et les discutons en regard des travaux antérieurs. Les implications théoriques, méthodologiques et pratiques de notre recherche sont envisagées.

\section{Connaître le sujet souffrant au travail : une perspective foucaldienne}

\section{Le sujet souffrant, effet d'un savoir}

Suivant ici Foucault $(1969 ; 1975 ; 1991)$, nombre de travaux en management étudient la manière dont des disciplines managériales (la gestion des ressources humaines, Townley, 1993 ; 1998 ; la comptabilité et le contrôle de gestion, Miller \& O’Leary, 1987 ; Ezzamel et al., 2008 ; le management stratégique, Knights \& Morgan, 1991 ; 1995 ; Ezzamel \& Willmott, 2010) nous constituent en tant que 'sujet'. Ce que nous pensons, disons, faisons, ne doit pas être vu comme le résultat de quelque essence, mais comme le produit de processus et de pratiques sociales qui se sont constitués historiquement. Et c'est au travers de ces pratiques et des rapports qu'elles tissent entre les hommes et le monde qui les entoure, que nous sommes fabriqués en tant que sujet. Trois grands types d'ensembles pratiques ont particulièrement retenu l'attention de Foucault $(1984,2001: 1395)$ :

- les pratiques discursives et les techniques de connaissances (de description, de classification, d'observation par exemple) par lesquelles nous cherchons à maîtriser 
les choses qui nous entourent et constituons un savoir sur celles-ci (Foucault, 1969).

Ces pratiques nous construisent à la fois en tant que sujet connaissant et à connaître.

- les pratiques d'organisation, de répartition dans l'espace et dans le temps, de surveillance, de punition ou de correction par lesquelles nous cherchons à infléchir la conduite des autres, pratiques qui participent d'un champ de pouvoir (Foucault, 1975 ; 1978) et nous constituent en tant que sujet de pouvoir.

- les pratiques ou techniques de soi par lesquelles nous nous travaillons et nous gouvernons nous-mêmes (Foucault, 1983 ; 1988). Ces pratiques participent d'un processus de subjectivation au travers duquel nous serions capables de nous constituer en tant que sujet éthique, c'est-à-dire sujet capable d'échapper aux relations de pouvoir et de savoir qui nous fabriquent en tant que sujet social.

Ce sont tout particulièrement les 'régimes' de savoir et de pouvoir qui ont retenu l'attention des chercheurs en management s'inscrivant dans cette perspective. Les savoirs produits par et pour le management des ressources humaines, analyse ainsi Townley (1993), ne sont pas neutres mais disposent d'un effet 'productif' et participent, en ce sens, de mécanismes de pouvoir. Le savoir, ici, est entendu comme un mécanisme d'assujettissement, autrement dit de fabrication d'un sujet : quelqu'un qui agit en accord avec les règles et les normes de ce savoir, endosse un rôle et occupe une 'position de sujet' particulière (Foucault, 1991), voire se définit lui-même dans les termes de ce savoir (Townley, 1993 ; Rose, 1999).

L'objet d'analyse, dès lors, est le 'caractère connaissable' (knowability, Townley, 1993 : 522 ) de l'individu : quels sont les processus qui permettent de connaître l'individu ? Comment ces processus participent-ils de la fabrication d'un 'sujet' particulier : du 'délinquant' (Foucault, 1975), du fou (Foucault, 1976a), de l'hystérique (Foucault, 1976b) ? Quels en sont les traits, contours et comportements? 
Rose (1999) a ainsi montré comment les connaissances développées en psychologie au cours $\mathrm{du} \mathrm{XX}^{\mathrm{e}}$ siècle -en particulier durant les deux premières guerres mondiales dans l'armée- ont progressivement colonisé l'entreprise, les relations entre l'État et la société au travers du 'Welfare State', et participé de la constitution d'un sujet moderne : un sujet 'libre', capable de 'choisir' et de se transformer lui-même. Parallèlement, les recherches critiques en management ont précisé les traits de ce sujet 'moderne' : un individu libre dont les objectifs doivent être orientés, via les processus budgétaires notamment, afin que ses comportements contribuent aux objectifs de l'organisation pour le contrôle de gestion (Miller \& O'Leary, 1987) ; un sujet 'rationnel', autonome, capable de prendre des risques et de maitriser, au moins en partie, l'environnement pour le management stratégique (Knights \& Morgan, 1991) ; un sujet 'comptable' de lui-même ('accountable', pour reprendre l'expression anglosaxonne, Townley, 1993 ; 1998) pour les ressources humaines, c'est-à-dire tout à la fois 'calculable' (i.e. dont on peut apprécier la valeur sur des dimensions par avance spécifiées), 'responsable' et capable de rendre compte (to account) de ses actions, de ses résultats et de lui-même.

Nous inscrivant dans cette perspective, nous considérons ainsi que le 'savoir' qui s'est progressivement constitué depuis la fin des années 1970 sur la souffrance au travail (le stress, l'épuisement professionnel, et ce qu'on appelle aujourd'hui les 'risques psycho-sociaux') amène à construire un 'sujet' particulier : un sujet qui entretient des relations spécifiques avec lui-même et le monde qui l'entoure, on le verra.

Cette perspective ne nie en rien le fait que, dans les organisations, des personnes souffrent au travail ; elle considère simplement que le mal ressenti est incommensurable aux 'mots' que nous utilisons pour le décrire, autrement dit, que le savoir qui se constitue sur le stress, le malêtre, l'épuisement professionnel au travail ne peut prétendre entièrement capturer la souffrance 'ressentie'. Au travers des concepts, des théories, des instruments de mesure qu'il 
déploie cependant, ce savoir promeut des règles et des normes de comportement (i. e. avoir des responsabilités, contrôler les évènements, faire preuve d'empathie vs. se tenir à distance, laisser-faire, avoir recours à la violence physique ou verbale), véhicule (et exclut) des notions (i. e. 'être stressé', 'pressurisé' vs. indifférent). Il constitue ainsi une 'grammaire’ par laquelle l'individu est susceptible de définir son identité sociale.

\section{Connaître le sujet souffrant : de l'intérêt des échelles d'auto-évaluation}

Quel sujet le savoir sur la souffrance au travail fabrique-t-il ? Quelles caractéristiques et normes de comportement appelle-t-il, occulte-t-il ? Répondre à cette question suppose de circonscrire et d'analyser ce savoir sur la souffrance au travail. Pour Foucault (1991), un savoir se définit comme une 'formation discursive', c'est-à-dire « un domaine pratique limité, qui a ses frontières, ses règles de formation et ses conditions d'existence » (p. 61), règles qui définissent «les choses qui peuvent être dites » (p. 63) à une époque donnée. Ces règles permettent de distinguer un savoir (médical par exemple), d'un autre savoir (sociologique par exemple) ou du sens commun.

Un savoir ne se résume cependant pas 'aux choses dites'. Un ensemble d'énoncés forme un savoir dans la mesure où certes, ils obéissent à des règles, mais également parce qu'ils sont liés à des formations 'extra-discursives'; un ensemble de conditions sociales et matérielles qui permettent ces énoncés. Ces dimensions extra-discursives renvoient notamment aux critères utilisés pour désigner ceux qui reçoivent le droit de tenir le discours (i. e. Qui a le droit de / est légitime pour parler de la souffrance au travail ?) ; L'échelle d'observation qui permet de désigner les objets du discours (i. e. Où regarde-t-on ? Comment ?) et «les modes d'enregistrement, de conservation, d'accumulation, de diffusion et d'enseignement [...] du discours » (Foucault, $1991: 67)$. 
Les savoirs sur la souffrance au travail dépendent ainsi de conditions (modalités d'organisation de l'espace, de coordination des activités notamment) et de dispositifs matériels (techniques d'examen par exemple) qui permettent à certains aspects d'être 'vus', de « venir à notre attention » (Lilley, 2001: 71), cependant que d'autres sont occultés, cachés. Les travaux s'inscrivant dans une perspective critique de la santé au travail se sont particulièrement attachés à étudier ce second aspect. Considérant les recherches et enquêtes menées sur la santé au travail par les organismes nationaux et internationaux notamment, ils dévoilent leurs angles morts, leurs 'invisibilités' : la non-reconnaissance ou reconnaissance tardive de certaines maladies professionnelles (Watterson, 1999), d'accidents du travail (Nichols, 1999), la négligence de facteurs sociopolitiques (interaction travail rémunéré travail domestique des femmes, situation familiale et isolement social éventuel, par exemple, Payne, 1999), économiques ou organisationnels (Nichols, 1999) essentiels dans la compréhension de la santé.

Les recherches en management, de leur côté, ont privilégié l'étude des 'visibilités', c'est-àdire de ce que les techniques d'enquêtes, d'enregistrements des données, d'organisation de l'espace notamment, permettaient de 'voir'. Dans cette perspective, Townley (1993) souligne le rôle fondamental des tests psychométriques, aux côtés des entretiens de sélection, dans la constitution du savoir sur les personnes dans les organisations. «Les tests de personnalité », relève-t-elle (p. 534) «permettent une standardisation de la personnalité, et les techniques des échelles introduisent les dimensions subjectives des individus dans la sphère de la connaissance et de la régulation », diffusant au passage l'idée qu'il existe en tout un chacun quelque chose comme une personnalité, dont les attributs peuvent être distingués, isolés, évalués (p. 535).

Les échelles de mesure de la souffrance présentent, à ce titre, de nombreux intérêts : 
- d'un point de vue empirique, la majeure partie des travaux de recherche publiés s'appuient sur ces outils plutôt que sur d'autres techniques d'étude (l'observation ou les entretiens en profondeur par exemple). Et nombre d'entreprises, en vue d'évaluer la souffrance au travail et de mettre en place des mesures préventives mènent des enquêtes qui reprennent tout ou partie de ces échelles.

- d'un point de vue théorique ensuite, ces échelles, à l'instar des tests psychométriques, procèdent de l'examen (Foucault, 1975 ; Townley, 1993) : une méthode d'observation scientifique qui permet de mesurer quantitativement les caractéristiques des individus (i. e. les différents aspects de leurs 'états' de souffrance et leurs déterminants), de les hiérarchiser, autrement dit de rendre visibles des différences en sorte que la souffrance, comme d'autres dimensions de l'individu (Townley, 1993 : 535), peut être 'calculée' et 'gérée'.

Les échelles de mesure de la souffrance constituent ainsi des techniques de visibilité qui participent pleinement du savoir scientifique sur la souffrance au travail.

En vue de définir plus avant les traits et caractéristiques du 'sujet souffrant', nous analysons en profondeur les échelles de mesure de la souffrance les plus couramment utilisées dans la recherche en management.

\section{Méthodologie}

\section{Choix des échelles de mesure de la souffrance au travail}

La souffrance au travail renvoie à différentes notions plus ou moins liées : stress, épuisement professionnel, mal-être, dépression (voir tableau 1 ci-après pour une définition des instruments et des construits mesurés). Chacune de ces notions a en outre été l'objet d'échelles particulières. Face à cette diversité, nous avons dû procéder à un choix. Nous nous sommes appuyés pour ce faire sur notre connaissance des recherches empiriques menées sur 
la souffrance au travail, mais également sur les indices de citation des échelles dans les travaux de recherche. Le tableau 1 ci-après présente ainsi, pour les échelles retenues, deux indices de citation : l'indice de Harzing qui recense le nombre de travaux disponibles sur GoogleScholar citant l'article de référence de l'échelle, d'un côté ; le nombre de citations de l'échelle dans des revues académiques disponibles dans la base Business Source Complete, de l'autre.

Deux échelles se sont naturellement imposées en raison de leur prédominance dans les études sur les situations de travail pour le compte d'entreprises ou d'institutions : les échelles de Karasek (JCQ) et de Siegrist (ERI). Si ces échelles ne mesurent pas le stress mais des facteurs de risque, elles sont utilisées et recommandées pour sa mesure (voir rapport Poisson, 2008).

L'échelle Perceived Stress Scale de Cohen, Kamarack et Mermerlstein (1983), dont la version française a été récemment validée est «largement utilisée et a fait l'objet de nombreuses études » (Bellinghausen, Collange, Botella, Emery, \& Albert, 2009: 366). Le Maslach Burnout Inventory fait aujourd'hui référence dans les études sur le syndrome d'épuisement professionnel (burnout). Les échelles HAD et GHQ, qui ont été construites afin d'identifier les patients souffrant de troubles psychiques dans des services de soins non psychiatriques, ont été utilisées en France notamment dans des collectes de données de grande ampleur auprès de salariés par des médecins du travail ${ }^{3}$. L'échelle SF-36, enfin, est une échelle de qualité de vie liée à la santé, qui a fait l'objet en 1998 de traductions et de validations dans 15 pays dans le cadre d'un projet international (voir Verger, Aulagnier, Schwoebel, \& Lang, 2005).

Nous avons fait le choix de ne pas présenter les résultats des analyses menées ${ }^{4}$ sur un certain

\footnotetext{
${ }^{3}$ Dans le cadre de l'Observatoire Médical du Stress de l'Anxiété et de la Dépression -OMSAD- de Renault pour HAD et dans le programme Santé Mentale Observatoire Travail Rhône-Alpes Centre, dit Samotrace, de l'Institut de Veille Sanitaire -InVS- pour GHQ.

${ }^{4}$ Les résultats sont disponibles auprès des auteurs.
} 
nombre d'échelles dont la version française avait été validée, et ce, en raison de la faiblesse de leurs indices de citation : l'échelle de Steffy \& Jones (1988), la mesure Shirom-Melamed Burnout de Shirom \& Melamed (2006) ou encore la mesure du Stress Psychologique de Lemyre \& Tessier (1988), disposent ainsi d'indices Harzing s'élevant respectivement à 54, 65 et 46 et d'indices BSC s'élevant respectivement à 4, 4 et 1 .

L’incidence de ces choix sur la validité des résultats sera discutée en conclusion. 
Tableau 1. Présentation des échelles d'auto-évaluation

\begin{tabular}{|c|c|c|c|c|c|c|}
\hline Sources & Source validation française & $\begin{array}{l}\text { Dénomination } \\
\text { et acronyme }\end{array}$ & Objectif de la mesure et contenu & $\begin{array}{l}\text { Nombre } \\
\text { d'items }\end{array}$ & $\begin{array}{l}\text { Indice* } \\
\text { de } \\
\text { citation } \\
\text { Harzing }\end{array}$ & $\begin{array}{l}\text { Indice* } \\
\text { de } \\
\text { citation } \\
\text { BSC*** }\end{array}$ \\
\hline $\begin{array}{l}\text { Cohen et al. } \\
\qquad(1983)\end{array}$ & Bellinghausen et al. (2009) & $\begin{array}{l}\text { Perceived Stress } \\
\text { Scale (PSS) }\end{array}$ & $\begin{array}{l}\text { Evaluer le degré de stress des situations. Les items mesurent } \\
\text { le degré selon lequel les individus trouvent leur vie } \\
\text { imprévisible, incontrôlable et surchargée. }\end{array}$ & 10 & 4618 & 50 \\
\hline $\begin{array}{l}\text { Zigmond \& } \\
\text { Snaith (1983) }\end{array}$ & $\begin{array}{l}\text { Lépine, Godchau, Brun, \& } \\
\text { Lempériere (1985) }\end{array}$ & $\begin{array}{c}\text { Hospital } \\
\text { Anxiety } \\
\text { Depression } \\
\text { Scale (HAD) }\end{array}$ & $\begin{array}{l}\text { Evaluer le niveau de symptomatologie dépressive et anxieuse } \\
\text { en éliminant les symptômes somatiques susceptibles de } \\
\text { fausser les évaluations chez des patients vus en médecine qui } \\
\text { présentent fréquemment des problèmes organiques. }\end{array}$ & 14 & 11185 & 108 \\
\hline $\begin{array}{l}\text { Ware \& } \\
\text { Sherbourne } \\
\quad(1992)\end{array}$ & $\begin{array}{l}\text { Leplege, Mesbas, \& Marquis } \\
\text { (1995) }\end{array}$ & $\begin{array}{l}\text { Medical } \\
\text { outcome Study } \\
\text { Short-Form } 36 \\
\quad(\text { SF-36) }\end{array}$ & $\begin{array}{l}\text { Evaluer la qualité de vie d'un individu, le degré de } \\
\text { répercussion d'un traumatisme sur la santé (troubles de santé } \\
\text { mentale) et sur le fonctionnement social ou au travail. Permet } \\
\text { d'évaluer également le besoin de soins des individus. }\end{array}$ & 36 & 15355 & 128 \\
\hline $\begin{array}{l}\text { Goldberg \& } \\
\text { Hillier (1979) }\end{array}$ & $\begin{array}{l}\text { Bolognini, Bettschart, } \\
\text { Zehndergubler, \& Rossier (1989); } \\
\text { Bettschart, Plancherel, \& } \\
\text { Bolognini (1991) }\end{array}$ & $\begin{array}{l}\text { General Health } \\
\text { Questionnaire } \\
\quad \text { (GHQ) }\end{array}$ & $\begin{array}{l}\text { Evaluer une souffrance psychique globale commune sans } \\
\text { présomption d'un diagnostic psychiatrique précis et de } \\
\text { mesures épidémiologiques. }\end{array}$ & 28 & 2479 & 311 \\
\hline $\begin{array}{l}\text { Maslach \& } \\
\text { Jackson } \\
(1981)\end{array}$ & $\begin{array}{c}\text { Girault (1989) } \\
\text { Girault-Lidvan (1996) }\end{array}$ & $\begin{array}{c}\text { Maslach } \\
\text { Burnout } \\
\text { Inventory (MBI) }\end{array}$ & $\begin{array}{l}\text { Evaluer les trois aspects de l'épuisement professionnel : } \\
\text { l'assèchement émotionnel, la dépersonnalisation, et le } \\
\text { sentiment de non accomplissement. }\end{array}$ & 22 & 2894 & 234 \\
\hline Siegrist (1996) & Niedhammer, et al., 1996 & $\begin{array}{c}\text { Effort Reward } \\
\text { Imbalance (ERI) }\end{array}$ & $\begin{array}{l}\text { Evaluer les facteurs de stress chroniques au travail. Le } \\
\text { déséquilibre entre les efforts personnels consentis et les } \\
\text { faibles récompenses reçues (pour ces efforts) produisent des } \\
\text { situations à risques. }\end{array}$ & 17 & 1770 & 158 \\
\hline Karasek, 1979 & $\begin{array}{l}\text { Niedhammer, Chastang, } \\
\text { Gendrey, David, \& Degioanni } \\
\text { (2006) }\end{array}$ & $\begin{array}{c}\text { Job Content } \\
\text { Questionnaire / } \\
\text { Job Demands } \\
\text { Control Support } \\
\text { Model } \\
\text { (JCQ ou JDC-S) }\end{array}$ & $\begin{array}{l}\text { Evaluer les caractéristiques sociales et psychosociales du } \\
\text { travail. La latitude décisionnelle, les exigences } \\
\text { psychologiques et le soutien social mesurent les tensions au } \\
\text { travail. D'autres aspects comme les exigences physiques et } \\
\text { l'insécurité au travail sont également mesurés. }\end{array}$ & 26 & 4750 & 157 \\
\hline
\end{tabular}

Business Source Complete de 1976 à 2011. 


\section{Analyse des échelles}

L'analyse des échelles retenues emprunte une démarche qualitative dite 'thématique'. L'ensemble des items ou questions composant les échelles ont été l'objet d'une catégorisation à un double niveau :

- Une catégorisation a priori (Huberman \& Miles, 1984) dans un premier temps. Nous avons dans ce cadre classé les items et questions selon qu'ils renvoyaient à l'une ou l'autre des catégories suivantes: 1/ rapport aux choses (i.e. événements, environnement de travail, récompenses notamment), 2/ rapport aux autres (i. e. collègues, supérieur hiérarchique, famille notamment), 3/ rapport à soi (i. e. sentiments, corps, énergie, fatigue, notamment); trois catégories renvoyant aux différents 'ensembles pratiques' distingués par Foucault (1984) dans son analyse de la construction du sujet. Rapidement cependant, il nous est apparu que ces catégories occultaient 4/ le rapport au temps d'un côté et 5/ le rapport à l'existence, de l'autre, deux dimensions qui, pour la première était présente dans l'ensemble des échelles étudiées et qui, pour la seconde distinguait en partie certaines échelles (HAD et GHQ en particulier) des autres en introduisant les idées de joie de vivre, d'espoir ou encore d'angoisse et de suicide, notions bien plus générales que celles portées par les autres catégories. Ces deux catégories (rapport au temps et rapport à l'existence) ont donc été ajoutées aux trois catégories initiales. Une fois le classement des items et questions stabilisé, chaque catégorie a été définie en vue de faciliter et de garantir la fiabilité des classifications opérées.

- Une sous-catégorisation a posteriori ensuite. En vue de préciser les dimensions caractéristiques de ces différentes catégories, nous avons ensuite, au sein de chacune des catégories, regroupé les items ou questions selon leur degré de similarité ou 
différence de sens (cf. Glaser \& Strauss, 1967). Ces différentes sous-catégories ont enfin été nommées en cherchant à rendre compte des caractéristiques ou traits de l’individu véhiculés par les items ou questions.

Cette double catégorisation a été effectuée tout d'abord indépendamment sur des sousensembles différents par les deux auteurs et un troisième chercheur. Ce partage des tâches permettait de vérifier que les trois dimensions retenues disposaient de définitions suffisamment claires pour permettre le classement d'items d'échelles différentes. C'est à cette occasion que les catégories de rapport au temps, d'un côté et de rapport à l'existence, de l'autre, ont été adjointes à celles initialement retenues. Ce partage des tâches garantissait également que les sous-catégories identifiées ne soient pas trop précocement regroupées et confondues, réduisant par là le risque de perdre les significations spécifiques portées par les items des différentes échelles. La catégorisation a ensuite été entièrement revue et corrigée par les trois chercheurs en vue de parvenir à un accord quant à la définition des catégories, des sous-catégories et les classements opérés. Si le classement des items dans les différentes catégories et leurs définitions n'ont posé aucune difficulté, des ajustements ont été opérés en vue d'harmoniser les dénominations des sous-catégories. Les désaccords sur ces dernières ont été résolus par discussion, le plus souvent en choisissant la dénomination la moins abstraite. La section suivante présente et commente les résultats obtenus.

\section{Résultats}

La présence ou absence des catégories dans les différentes échelles témoignent de leurs objectifs (voir Tableau 2 ci-dessous). Les échelles JCQ et ERI comportent des items évoquant les rapports au temps, aux choses et aux autres mais pas les rapports à soi et à l'existence, ce qui reflète leur destination : mesurer le niveau de risque de la situation de travail, et non le niveau de stress ou de mal-être. Les échelles HAD et GHQ, de leurs côtés, présentent des 
items relevant du rapport à l'existence, et ce, dans des proportions importantes (la moitié des items pour HAD, le tiers pour GHQ). Les échelles HAD et SF-36, enfin, ne présentent pas d'items relevant du rapport aux choses. Dans ces deux échelles prédominent des items relatifs au rapport à soi. Ces caractéristiques reflètent les objectifs de ces outils, principalement destinés à opérer un diagnostic de la santé psychique de l'individu.

Tableau 2. Présence des catégories dans les échelles étudiées

\begin{tabular}{|c|c|c|c|c|c|c|}
\hline $\begin{array}{c}\text { Présence } \\
\text { dans } \\
\text { l'échelle du } \\
\text { rapport... }\end{array}$ & au temps & $\begin{array}{c}\text { aux } \\
\text { choses }\end{array}$ & aux autres & à soi & $\begin{array}{c}\text { à } \\
\text { l'existence }\end{array}$ & $\begin{array}{c}\text { Nombre } \\
\text { de } \\
\text { catégories } \\
\text { présentes }\end{array}$ \\
\hline PSS & $\mathrm{x}$ & $\mathrm{x}$ & & $\mathrm{x}$ & & $3 / 5$ \\
\hline HAD & $\mathrm{x}$ & & & $\mathrm{x}$ & $\mathrm{x}$ & $3 / 5$ \\
\hline SF-36 & $\mathrm{x}$ & & $\mathrm{x}$ & $\mathrm{x}$ & & $3 / 5$ \\
\hline GHQ & $\mathrm{x}$ & $\mathrm{x}$ & & $\mathrm{x}$ & $\mathrm{x}$ & $4 / 5$ \\
\hline MBI & $\mathrm{x}$ & $\mathrm{x}$ & $\mathrm{x}$ & $\mathrm{x}$ & & $4 / 5$ \\
\hline JCQ & $\mathrm{x}$ & $\mathrm{x}$ & $\mathrm{x}$ & & & $3 / 5$ \\
\hline ERI & $\mathrm{x}$ & $\mathrm{x}$ & $\mathrm{x}$ & & & $3 / 5$ \\
\hline
\end{tabular}

Dans les paragraphes qui suivent, nous définissons chacune de ces catégories et les dimensions les composant.

\section{Rapport au temps}

Cette catégorie regroupe un ensemble de questions ou d'items au travers duquel l'individu est invité, directement ou indirectement à rendre compte de son état, à apprécier son environnement ou encore ses relations aux autres, et ce dans le temps. Toutes les échelles étudiées appellent une 'évaluation temporelle' de la part du répondant. Cette appréciation temporelle se décline au travers de trois dimensions (voir tableau 3) principales : 
- Une fréquence sur une période donnée, qu'il s'agisse d'une période courte et rapprochée (une semaine, un mois) ou d'une période plus longue (un an) ;

- Une comparaison de la situation actuelle vécue avec un passé non précisé : un 'avant', 'd'habitude' ;

- Une évaluation de la contrainte ou de la pression que représente 'le temps'.

Tableau 3. Sous-catégorisation des questions ou items indiquant un rapport au temps

\begin{tabular}{|l|l|}
\hline \multicolumn{1}{|c|}{ Sous-catégories } & \multicolumn{1}{c|}{ Exemples } \\
\hline $\begin{array}{l}\text { Capacité d'appréciation d'une fréquence } \\
\text { (relativement à la semaine, au mois, à } \\
\text { l'année écoulée) }\end{array}$ & $\begin{array}{l}\text { PSS (Cohen et al., 1983). Les 10 questions sont } \\
\text { formulées de la manière suivante : } \\
\text { Au cours du mois précédent, à quelle fréquence avez- } \\
\text { vous .. ? } \\
\text { Les items de réponse proposés se répartissent ainsi : } \\
\text { 0- Jamais } \\
\text { 1. Presque jamais } \\
\text { 2. Quelquefois } \\
\text { 3. Assez souvent } \\
\text { 4. Très souvent }\end{array}$ \\
\hline $\begin{array}{l}\text { Capacité à comparer une situation ou une } \\
\text { fréquence avec le passé }\end{array}$ & $\begin{array}{l}\text { 1'année dernière à la même époque, comment trouvez- } \\
\text { vous votre état de santé en ce moment ? } \\
\text { - Bien meilleur que l'an dernier/plutôt meilleur/ }\end{array}$ \\
\hline $\begin{array}{l}\text { Capacité à apprécier la pression du temps pareil/ plutôt moins bon/beaucoup } \\
\text { moins bon }\end{array}$ \\
\hline $\begin{array}{l}\text { ERI (Siegrist, 1996). Le répondant exprime son accord } \\
\text { ou désaccord avec les affirmations proposées, et } \\
\text { indique éventuellement le degré de perturbation } \\
\text { associé à cette attitude («pas du tout perturbé(e)» à } \\
\text { «très perturbé(e)») }\end{array}$ \\
$\begin{array}{l}\text { Je suis constamment pressé(e) par le temps à cause } \\
\text { d'une forte charge de travail } \\
\text { Je suis souvent contraint(e) à faire des heures } \\
\text { supplémentaires }\end{array}$ \\
\hline
\end{tabular}

Plus marginalement (1 question), le temps est envisagé comme un indicateur de réalisation des activités (GHQ).

Sous ces différents aspects, les échelles supposent que l'individu tienne une forme de comptabilité des évènements qui l'affectent et de ses états, de sorte qu'il soit capable de 
rendre compte de leur fréquence sur une période donnée, de comparer cette dernière avec une situation antérieure et d'en apprécier le poids ('la pression' du temps). Le temps est ainsi indirectement compris comme une dimension objective dans laquelle on peut facilement se positionner (la semaine, le mois, l'année écoulée), que l'on peut soupeser (au travers d'une fréquence, de la pression ou de la ressource qu'il représente), données que l'on est capable d'enregistrer et de restituer.

Prises ensemble, ces caractéristiques du temps et de l'individu supposent des capacités cognitives (enregistrer l'information, la traiter, la restituer) et évaluatives également très présentes dans les items ou questions relevant des 'relations aux choses' et aux 'autres'.

\section{Rapport aux choses}

Cette catégorie regroupe un ensemble de questions ou d'items au travers duquel l'individu est invité à évaluer des évènements, des aspects de son environnement de travail ou son travail lui-même, ces différents aspects étant définis en termes généraux (i.e. 'les choses importantes de votre vie' dans le PSS par exemple) ou précis (i. e. 'mes perspectives de promotion' dans le ERI par exemple). Les échelles ayant pour objet d'appréhender directement ou indirectement la souffrance au travail (PSS, MBI, ERI et JCQ) comportent toutes des items ou questions supposant pour l'individu de rendre compte de son 'rapport aux choses'. Parmi les échelles développées par les psychologues ou psychiatres pour évaluer un état de souffrance psychique (HAD) ou de santé générale (SF-36, GHQ), seul le questionnaire GHQ comporte des items appartenant à cette dimension.

Lorsqu'elle est présente, l'appréciation du rapport qu'entretient l'individu avec les choses se décline sous quatre dimensions (voir tableau 4) :

- Une évaluation des exigences du travail (charge, degré de nouveauté ou de routine, niveau de concentration, d'efforts, de compétences ou de responsabilité impliqués) et des marges de liberté ou d'autonomie associée (ERI, JCQ); 
- Une évaluation du travail réalisé ou des efforts fournis (GHQ, MBI) ;

- Une évaluation des récompenses matérielles ou symboliques reçues en regard des efforts fournis (ERI, JCQ) ;

- Une capacité à maîtriser ou contrôler les évènements (PSS) voire les prédire (ERI, GHQ).

Tableau 4. Catégorisation des questions ou items indiquant un rapport aux choses

\begin{tabular}{|c|c|}
\hline Sous-catégories & Exemples \\
\hline $\begin{array}{l}\text { Capacité à évaluer les exigences du } \\
\text { travail et les marges de liberté associées }\end{array}$ & $\begin{array}{l}\text { JCQ (Karasek, 1979) } \\
\text { - Mon travail me permet de prendre souvent des décisions } \\
\text { moi-même } \\
\text { - Dans ma tâche, j'ai très peu de libertés pour décider } \\
\text { comment je fais mon travail } \\
\text { 1. Pas du tout d'accord / 2. Pas d'accord/ 3. D'accord/ } 4 . \\
\text { Tout à fait d'accord }\end{array}$ \\
\hline $\begin{array}{l}\text { Capacité à évaluer le travail réalisé et les } \\
\text { efforts fournis }\end{array}$ & $\begin{array}{l}\text { GHQ (Goldberg \& Hillier, 1979) - Avez-vous eu le } \\
\text { sentiment que dans l'ensemble vous faisiez bien les } \\
\text { choses? } \\
\text { - Avez-vous été satisfait(e) de la façon dont vous avez fait } \\
\text { votre travail/vous vous êtes acquitté de votre tâche? } \\
\text { 0. Plus satisfaite/0. Comme d'habitude /1. Moins } \\
\text { satisfaite que d'habitude / 1. Bien moins satisfaite }\end{array}$ \\
\hline $\begin{array}{l}\text { Capacité à évaluer les récompenses } \\
\text { matérielles et symboliques en regard des } \\
\text { efforts fournis }\end{array}$ & $\begin{array}{l}\text { MBI (Maslach \& Jackson, 1981). Je me sens frustré(e) par } \\
\text { mon travail. } \\
\text { 1. Jamais } \\
\text { 2. Quelques fois par an } \\
\text { 3. Une fois par mois } \\
\text { 4. Quelques fois par mois } \\
\text { 5. Une fois par semaine } \\
\text { 6. Quelques fois par semaine } \\
\text { 7. Tous les jours }\end{array}$ \\
\hline $\begin{array}{l}\text { Capacité à maîtriser ou contrôler les } \\
\text { évènements }\end{array}$ & $\begin{array}{l}\text { PSS (Cohen et al., 1983). Au cours du mois précédent, à } \\
\text { quelle fréquence: } \\
\text { - Avez-vous été dérangé(e) par un évènement inattendu? } \\
\text { - Vous a-t-il semblé difficile de contrôler les choses } \\
\text { importantes de votre vie? } \\
\text { - Avez-vous senti que les choses allaient comme vous } \\
\text { vouliez? }\end{array}$ \\
\hline
\end{tabular}

Ces dimensions supposent que l'individu soit capable de percevoir et d'évaluer au travers de caractéristiques précises son environnement de travail et les évènements qui l'affectent. Ces aspects sont envisagés comme extérieurs à l'individu, extériorité avec laquelle il entretient un 
rapport 'transactionnel' : il en évalue les exigences, apprécie l'effort qu'il fournit, les 'récompenses' reçues en regard de ces efforts. La notion de frustration, qui suppose que l'individu voit dans son travail un moyen de réaliser certaines de ses aspirations, aspirations dès lors différentes des 'exigences' du travail, n'est mentionnée que par le MBI. En d'autres termes, le travail n'est pas envisagé, dans la plupart des échelles étudiées, comme un moyen de 'se réaliser', contrairement aux idées généralement véhiculées par les doctrines managériales.

Pour autant, ce rapport aux choses n'est pas nécessairement ou complètement subi. Les questions proposées dans l'échelle PSS, et, plus marginalement, certaines de celles de l'échelle d'ERI, supposent que l'individu dispose d'une capacité plus ou moins importante, si ce n'est de contrôler ou maîtriser les évènements extérieurs, à tout le moins de les prédire. Ceci ne signifie cependant pas que l'individu puisse avoir 'prise' ou influencer de quelque manière que ce soit les 'exigences' de son travail. Celles-ci s'expriment au travers de caractéristiques qui apparaissent comme désincarnées, indépendantes de toute relation hiérarchique. Il ne s'agit ainsi pas de satisfaire la demande des supérieurs (ou du supérieur) ou de collègues mais de faire face à une 'charge', des 'responsabilités ', des 'choses'.

\section{Rapport aux autres}

Les items ou questions relatifs au rapport aux autres témoignent également de la prégnance d'une relation de type transactionnel. Les items ou questions regroupés dans cette catégorie renvoient à une appréciation par l'individu de ses relations avec autrui, qu'elles soient envisagées comme un facteur de souffrance ou mal-être ou comme une 'variable' affectée par l'état de souffrance de l'individu. Cette dimension n'est pas présente dans les échelles HAD, GHQ et PSS alors qu'elle est importante dans les échelles MBI, ERI, JCQ et SF-36. Dans une 
perspective très proche de celle caractérisant le 'rapport aux choses', le rapport aux autres se décline au travers des dimensions suivantes (voir tableau 5):

- Une évaluation du travail d'autrui, de ses actions et de ses compétences ;

- Une évaluation de l'incidence du travail réalisé ou de l'état de l'individu sur autrui (GHQ, MBI) ;

- Une évaluation des 'récompenses reçues' par l'individu en regard de l'effort fourni. Cet aspect est appréhendé au travers du soutien ou du respect dont le supérieur ou les collègues témoignent à l'endroit de l'individu (ERI, JCQ) ou au travers d'un gain ou d'une perte d'énergie qu'il subit dès lors qu'il 'travaille avec ou pour des gens' (MBI).

Tableau 5. Catégorisation des questions ou items indiquant un rapport aux autres

\begin{tabular}{|l|l|}
\hline \multicolumn{1}{|c|}{ Sous-catégories } & \multicolumn{1}{|c|}{ Exemples } \\
\hline $\begin{array}{l}\text { Capacité à évaluer le travail d'autrui, ses } \\
\text { actions et ses compétences }\end{array}$ & $\begin{array}{l}\text { JCQ (Karasek, 1979) } \\
- \text { Attendre le travail de collègues ou d'autres } \\
\text { départements ralentit souvent mon propre travail } \\
\text { - Les collègues avec qui je travaille sont des gens } \\
\text { professionnellement compétents }\end{array}$ \\
\hline $\begin{array}{l}\text { Capacité à évaluer l'incidence du travail } \\
\text { réalisé ou de l'état de l'individu sur autrui }\end{array}$ & $\begin{array}{l}\text { MBI (Maslach \& Jackson, 1981) } \\
\text { Je sens que je m'occupe de certains } \\
\text { patients/clients/élèves de façon impersonnelle comme } \\
\text { s'ils étaient des objets } \\
- \text { Je suis devenu(e) plus insensible aux gens depuis } \\
\text { que j'ai ce travail }\end{array}$ \\
\hline $\begin{array}{l}\text { Capacité à évaluer les 'récompenses reçues' } \\
\text { par l'individu en regard de l'effort fourni }\end{array}$ & $\begin{array}{l}\text { ERI (Siegrist, 1996) } \\
- \text { Je reçois le respect que je mérite de la part de mes } \\
\text { supérieurs } \\
- \text { Je reçois le respect que je mérite de la part de mes } \\
\text { collègues }\end{array}$ \\
\hline
\end{tabular}

Au travers de ces différents aspects, le rapport aux autres qu'entretient l'individu est envisagé comme une transaction, voire une forme d'investissement : ce que l'individu 'fournit' au travers de son travail aura une incidence sur 'autrui' qu'il s'agisse d'un collègue de travail ou 
d'un usager/client, incidence dont il attend un 'retour', retour qu'il est susceptible de mesurer en termes de gain/perte d'énergie, de soutien ou de reconnaissance.

À la différence du 'rapport aux choses', ce 'rapport aux autres' n'induit cependant pas quelque capacité à maîtriser ou prédire le comportement d'autrui ; de même qu'il ne véhicule pas, dans la plupart des échelles, d'exigences (en termes de charges, responsabilités spécifiques par exemple). Seule l'échelle MBI suppose que l'individu 'prenne en charge efficacement' les problèmes d'autrui et fasse preuve d'empathie ou de compréhension à son endroit. Et si l'échelle JCQ évoque bien 'des ordres' de la part de la hiérarchie, ce n'est pas tant pour souligner les exigences qu'ils véhiculent que leurs contradictions éventuelles et leurs incidences sur l'autonomie.

\section{Rapport à soi}

La catégorie 'rapport à soi' regroupe un ensemble d'items ou questions au travers duquel l'individu est invité à rendre compte de 'lui-même'. En dehors des échelles JCQ et ERI, qui ont plus pour objet de rendre compte des facteurs de souffrance au travail que de cette souffrance elle-même, toutes les échelles étudiées appellent le répondant à dire 'son état', l'invitant par là à exprimer cette souffrance et/ou en apprécier les symptômes.

De manière récurrente, rendre compte de son 'état' suppose pour l'individu d'évaluer les dimensions suivantes (voir tableau 6) :

- Son niveau d'énergie ou de fatigue,

- Son niveau d'activité,

- Son niveau de tension ou d'énervement,

- Ses émotions (tristesse par exemple) ou sa capacité à les maitriser,

- Son état de santé,

Et d'évaluer les incidences de ses différents 'niveaux' sur sa capacité à réaliser des activités. 


\section{Tableau 6. Catégorisation des questions ou items indiquant un}

rapport à soi

\begin{tabular}{|l|l|}
\hline \multicolumn{1}{|c|}{ Sous-catégories } & \multicolumn{1}{c|}{ Exemples } \\
\hline $\begin{array}{l}\text { Capacité à rendre compte de son niveau } \\
\text { d'énergie ou de fatigue }\end{array}$ & $\begin{array}{l}\text { MBI (Maslach \& Jackson, 1981). } \\
\text { - Je me sens plein(e) d'énergie } \\
\text { - Je me sens au bout du rouleau } \\
\text { - Je me sens émotionnellement vidé(e) par mon travail }\end{array}$ \\
\hline $\begin{array}{l}\text { Capacité à rendre compte de son niveau } \\
\text { d'activité }\end{array}$ & $\begin{array}{l}\text { SF 36 (Ware \& Sherbourne, 1992). Au cours des } 4 \\
\text { dernières semaines, en raison de votre état physique : } \\
\text { - Avez-vous réduit le temps passé à votre travail ou à } \\
\text { vos activités habituelles } \\
\text { - Avez-vous fait moins de choses que ce que vous } \\
\text { auriez souhaité ? }\end{array}$ \\
\hline $\begin{array}{l}\text { Capacité à rendre compte de son niveau } \\
\text { de tension ou d'énervement }\end{array}$ & $\begin{array}{l}\text { HAD (Zigmond \& Snaith, 1983) } \\
\text { - Je me sens tendu(e) ou énervé(e) } \\
\text { - J'éprouve des sensations de peur et j'ai l'estomac } \\
\text { noué } \\
\text { - J'ai la bougeotte et n'arrive pas à tenir en place }\end{array}$ \\
\hline $\begin{array}{l}\text { Capacité à rendre compte de ses } \\
\text { émotions }\end{array}$ & $\begin{array}{l}\text { SF 36 (Ware \& Sherbourne, 1992). } \\
\text { Au cours des dernières semaines y a-t-il eu des } \\
\text { moments où } \\
\text { - Vous vous êtes senti(e) si découragé(e) que rien ne } \\
\text { pouvait vous remonter le moral ? } \\
\text { - Vous vous êtes senti(e) triste et abattu(e) ? }\end{array}$ \\
\hline $\begin{array}{l}\text { GHQ (Goldberg \& Hillier, 1979). Récemment } \\
\text { santé }\end{array}$ & $\begin{array}{l}\text { - Vous êtes-vous senti (e) parfaitement bien et en } \\
\text { bonne santé ? } \\
\text { - Avez-vous éprouvé le besoin d'un bon remontant ? }\end{array}$ \\
\hline
\end{tabular}

Comme pour les catégories précédentes, le 'rapport à soi' suppose pour l'individu d'être capable d'évaluation, évaluation qui porte en l'occurrence ici sur les différentes dimensions de ce qu'il est. Le rapport à soi appelle donc l'individu à une forme de réflexivité sur ce qu'il 'est' et ce qu'il 'fait', autrement dit qu'il se prenne lui-même comme objet d'évaluation. Les échelles n'appellent cependant pas tant à l'introspection qu'à un détachement, une objectivation de soi, en ce qu'il s'agit de s'apprécier au travers de dimensions qui s'imposent (et non d'errer dans les tréfonds de l'âme). Le 'soi' dont il est question ici est composé d'un corps dont on peut apprécier l'état de santé, le niveau de tension, d'énergie et d'activité. Ce 'soi' comporte également une dimension 'émotionnelle', dimension qu'il s'agit le plus 
souvent de maîtriser ou recouvrer (confiance en soi, bonne humeur), en ce qu'elle est susceptible d'influencer le niveau d'activité.

L'individu, comme l'environnement, n'est ainsi pas envisagé comme le 'tout' auquel renvoie l'étymologie, mais comme un ensemble de dimensions que l'on peut apprécier indépendamment les unes des autres. Ce qui toutefois paraît importer, c'est la mesure avec laquelle les différents 'niveaux' dont témoignent ces dimensions ont une influence sur l'énergie et l'activité de l'individu. Autrement dit ici, la 'souffrance' n'importe pas pour ellemême, mais pour ses incidences sur le travail.

Cette conception du sujet dont témoigne le rapport à soi tranche avec celle véhiculée dans le 'rapport à l'existence'.

\section{5. Rapport à l'existence}

Sont ici regroupés l'ensemble des items ou questions qui appellent l'individu à apprécier sa relation à la vie en général. Seules les échelles HAD et GHQ comportent de tels aspects, les autres échelles appelant plus volontiers une appréciation d'un rapport à des aspects spécifiés (un autre, qu'il soit client, collègue, supérieur ; une 'chose', qu'il s'agisse d'un évènement, d'un travail, d'une récompense par exemple). Lorsqu'il est présent, ce rapport à l'existence se décline au travers des dimensions suivantes :

- Prendre du plaisir,

- Ressentir des peurs, des soucis, des angoisses sans qu'ils soient définis,

- Espérer,

- Penser que l'on ou que la vie vaut quelque chose, ce qui, dans le cas contraire, peut conduire à des tentations suicidaires. 
Le rapport à l'existence se définit ainsi par des orientations générales comme l'espoir, le plaisir, le sentiment d'utilité ou la peur, sans que ces orientations n'aient d'objet ou que leurs incidences ne soient prises en compte (en dehors de la mort). La dimension évaluative, prégnante dans les autres catégories analysées, perd quelque peu de son emprise. Comme les rapports à soi, au temps, aux autres et à l'environnement, le rapport à l'existence n'est pas envisagé comme un tout mais au travers de différentes composantes.

Tableau 7. Catégorisation des items ou questions relevant du rapport à l'existence

\begin{tabular}{|l|l|}
\hline \multicolumn{1}{|c|}{ Sous-catégories } & \multicolumn{1}{c|}{ Exemples } \\
\hline $\begin{array}{l}\text { Capacité à rendre compte d'un état de } \\
\text { plaisir }\end{array}$ & $\begin{array}{l}\text { HAD (Zigmond \& Snaith, 1983). } \\
\text { - Je ris et je vois le bon côté des choses } \\
\text { - Je me réjouis à l'idée de faire certaines choses }\end{array}$ \\
\hline $\begin{array}{l}\text { Capacité à rendre compte d'angoisses, de } \\
\text { peur }\end{array}$ & $\begin{array}{l}\text { HAD (Zigmond \& Snaith, 1983). } \\
\text { - J'éprouve des sensations soudaines de panique } \\
\text { - Je me fais du souci }\end{array}$ \\
\hline $\begin{array}{l}\text { Capacité à rendre compte de la valeur de } \\
\text { l'existence ou de sa propre valeur }\end{array}$ & $\begin{array}{l}\text { GHQ (Goldberg \& Hillier, 1979). Récemment : } \\
\text {-Vous êtes-vous considéré(e) comme quelqu'un qui ne } \\
\text { valait rien? } \\
\text {-Avez-vous eu le sentiment de jouer un rôle utile dans la } \\
\text { vie ? }\end{array}$ \\
\hline Capacité à rendre compte d'espoirs & $\begin{array}{l}\text { GHQ (Goldberg \& Hillier, 1979). Récemment } \\
\text { - Avez-vous eu le sentiment que la vie est totalement } \\
\text { sans espoir? }\end{array}$ \\
\hline
\end{tabular}

\section{Discussion et conclusion}

Nous reprenons ici les résultats principaux de la recherche, apprécions leur validité, les discutons en regard des travaux antérieurs, et envisageons quelques voies de recherche qu'il nous semble fructueux de poursuivre.

Notre recherche avait pour objectif de circonscrire les traits du sujet que construisent les méthodes d'investigation de la souffrance au travail. En étudiant ce que voient les techniques d'auto-évaluation de la souffrance, notre recherche complète les travaux s'inscrivant dans une perspective critique de la santé au travail, recherches qui se sont essentiellement attachées à dévoiler les invisibilités des recherches scientifiques (enquêtes épidémiologiques notamment). 


\section{Un individu calculable}

En premier lieu, les résultats mettent en évidence que les échelles d'évaluation de la souffrance au travail, à l'instar d'autres techniques d'étude des personnes dans les organisations, véhiculent l'idée d'un individu calculable (Townley, 1993). Plus précisément, les catégories relevant du rapport à soi et du rapport à l'existence tendent à décomposer l'individu et sa souffrance en un ensemble de manifestations (corporelle, émotionnelle, énergétique principalement pour le rapport à soi) ou de dimensions (plaisir, anxiété, évaluation de la 'valeur' de la vie et de soi-même dans le rapport à l'existence).

\section{Un individu comptable}

En second lieu, les catégories relevant du rapport au temps, aux choses et aux autres, témoignent d'un individu 'comptable', confirmant ici les travaux de Pezet (2007: 7) à la suite de Hoskin et Macve (1986). Si la notion d'accountability est classiquement comprise comme impliquant la capacité de l'individu à rendre compte et expliquer son comportement, ses attitudes ou ses résultats, notre recherche en révèle une acception plus littérale.

En dehors des échelles HAD et SF-36 (dans lesquelles le rapport aux choses n'est pas envisagé), l'individu susceptible d'être stressé ou troublé psychiquement est un individu qui observe son environnement, en soupèse et enregistre les différents aspects. Il doit être capable de mobiliser sa mémoire et ses capacités cognitives pour se souvenir, comparer sa situation actuelle à celle passée ou apprécier une fréquence (JCQ, ERI).

Cet 'œil comptable' n'est pas que tourné vers l'extérieur, mais également vers l'individu luimême : il est à l'écoute et enregistre les manifestations de son corps, des pensées qui le traverse, de ses émotions; il peut se rappeler ses états antérieurs, les comparer à son état présent, rendre compte de leur fréquence voire envisager leur interdépendance (i. e. 
l'incidence de son état émotionnel, par exemple, sur son niveau d'énergie disponible). Le rapport à soi induit ici par les échelles d'auto-évaluation de la souffrance suppose, comme d'autres techniques de développement personnel (voir Townley, 2002: 274-75), que l'individu s'extrait de lui-même pour se regarder comme un objet et en dire la 'vérité'. Bien que privilégiant un 'rapport à l'existence', les échelles HAD et GHQ supposent une même distanciation : il s'agit de rendre compte d'angoisses, joies, idées suicidaires, autrement dit de décomposer sa relation à la vie.

Si l'on excepte l'échelle HAD (dans laquelle le rapport aux autres est absent), cette comptabilité permet à l'individu de comparer ce qu'il reçoit de l'environnement (de l'organisation, de ses collègues ou de son supérieur) avec les efforts qu'il fournit. La relation qu'il entretient avec le monde est ainsi essentiellement instrumentale, transactionnelle. L'individu développe des attentes par rapport aux autres et interagit principalement au travers de l'évaluation.

Qu'ils aient trait à la classification a priori ou à des sous-catégories élaborées via la comparaison des items des questionnaires, les résultats ne sont pas modifiés lorsque :

1/ Les échelles du stress au travail de Steffy \& Jones (1988), la mesure Shirom-Melamed Burnout de Shirom \& Melamed (2006) et celle du Stress Psychologique de Lemyre \& Tessier (1988) sont adjointes : leurs items renvoient aux cinq catégories identifiées et peuvent être classés dans des sous-catégories dont les significations sont soit identiques soit très proches de celles ici rapportées ;

2/ Les échelles JCQ/JDSC et ERI sont enlevées : les sous-catégories portant sur l'environnement de travail en effet, leur sont certes propres dans le cadre des résultats ici rapportés, mais apparaissent également dans d'autres échelles (l'échelle de Steffy \& Jones, 1988, notamment). 
Ces éléments et analyses complémentaires invitent à conclure à la bonne validité des analyses menées.

\section{De l'emprise d'une rationalité économique}

Sous ces différents angles, la 'comptabilité' dont il est question ici s'inscrit dans la rationalité économique qui, selon Townley (2002), caractérise nombre de théories orthodoxes en management : l'individu semble pris dans une causalité, qui, bien que relevant de mécanismes psychologiques, n'en est pas moins mécanique. C'est parce qu'il évalue négativement son environnement et/ou ce qu'il reçoit qu'il souffre. Et, s'il entretient des relations avec des 'collègues', sa rationalité semble abstraite, des-enchâssée, exempte de toute influence culturelle ou sociale.

Si Rose (1999) avait mis en évidence la colonisation de la psychologie dans les domaines du management des personnes dans l'entreprise, l'armée, l'éducation et sa participation à la conception du Welfare State, les résultats de la recherche pointent que les hypothèses managériales et économiques (idée de 'rationalité' et de recherche de satisfaction d'un intérêt) ont également 'colonisé' les théories psychologiques de la souffrance. Cette empreinte de l'économique sur le psychologique est également très présente dans la manière dont la souffrance est appréhendée. Dans la plupart des échelles étudiées en effet, ce n'est pas tant la souffrance qui importe que ses incidences sur le niveau d'énergie ou d'activités de l'individu (i. e. Peut-il faire plus, autant, ou moins qu'avant? Est-il dans l'action ou pas ?). Son niveau d'activité témoigne de sa présence et de sa contribution au monde dans lequel il évolue.

Cette prégnance des hypothèses économiques orthodoxes sur la conception du sujet portée par les échelles étudiées vient appuyer l'invite de Duncan (2007 : 205) à ne pas considérer que seules les structures de surveillance mises en place par les institutions médicales participent des relations de pouvoir et de savoir sur la santé. «Après tout », souligne-t-il (Duncan, 2007 : 
205, notre traduction), « dès lors que l'on envisage le pouvoir en termes foucaldiens comme un moyen au travers duquel des relations s'expriment comme quelque chose qui circule et non comme quelque chose de fixe, nous devons porter attention à tous ceux qui sont impliqués dans les relations médicales et de soin ».

En troisième lieu, nos résultats montrent que quoique rationnel et actif, le sujet construit par les échelles d'évaluation n'est pas un 'acteur'. Au travers de ses capacités comptables, l'individu est susceptible de prédire et contrôler (au sens de surveiller) les événements et le monde qui l'entoure (notamment dans les échelles JCQ, PSS, ERI). Mais il ne peut, par son action ou son comportement, en modifier le cours et participer ainsi de la construction sociale de son environnement de travail : il doit s'adapter, trait derrière lequel on peut voir la marque d'une conception disciplinaire, normalisatrice du travail (Foucault, 1975). Cette dimension est également véhiculée par les différents comportements que les questions ou items suggèrent (et excluent) : l'individu doit témoigner de compréhension à l'endroit des clients ou usagers avec lesquels il est en contact et non les mettre à distance (i. e. échelle MBI), attendre des récompenses 'symboliques' et non seulement un salaire (ERI, JCQ), s'adapter et non contempler ou chercher à changer le monde, 'souffrir' et non se révolter, par exemple.

Ces résultats, qui portent sur les techniques de visibilité du sujet, complètent ceux des travaux portant sur les discours et les doctrines managériales. À la suite de Foucault (2004) et de Rose (1999), certains travaux en management (voir notamment Miller \& O’Leary, 1987 ; Knights \& Morgan, 1991 ; 1995 ; Oakes, Townley, \& Cooper, 1998) ont souligné la convergence de ces discours et doctrines managériaux autour de l'idée d'un sujet libre, autonome, capable de choisir et de 'se réaliser'. Parallèlement, Duncan (2007) a montré combien les discours sur la santé l'envisagent comme une capacité à «faire ce que l'on peut de nos vies », «nous développer» (p. 153), à «travailler à la réalisation de nos [ses] potentialités biologiques et de celles que nous [il] aurons[a] définies de manière réaliste » (p. 9), autrement dit de 
«fonctionner comme un Homme, c'est-à-dire de manière libre et autonome » (p. 160, italiques par nos soins).

Ces idées d'autonomie, de liberté et de réalisation de soi qui marquent les discours du management et de la santé font écho à la figure de l'individu 'entrepreneur de lui-même' mise en évidence par Michel Foucault $(1978,2004)$ dans son analyse des théories économiques néolibérales : un individu avant tout constitué d'un capital, lui-même défini par un ensemble de traits innés et acquis, qu'il ne peut vouloir que faire fructifier, 'réaliser' en vue d'obtenir un 'retour sur investissement'.

Cette idée d'un individu-entreprise apparaît, de prime abord, cohérente avec celle de l'individu 'comptable' mise en évidence dans le cadre de notre analyse. Pour être 'entrepreneur de soi-même' en effet, il faut bien être capable de décomposer, enregistrer, et d'évaluer ce capital que l'on est, d'apprécier les investissements réalisés (les 'efforts fournis') et ce que l'on en 'retire' (les récompenses, le 'bénéfice' reçus).

Cette convergence entre discours managériaux et techniques de visibilité de la souffrance s'arrête cependant là. Alors que l'entrepreneur de lui-même porté par les discours managériaux dispose de désirs et d'objectifs qui lui sont propres (Miller \& O’Leary, 1987 ; Rose, 1999), le sujet construit par les techniques de mesure étudiées est un individu qui doit avant tout s'adapter à des exigences qui lui sont extérieures. Il ne peut vouloir autre chose que ce que l'on attend de lui (être 'compréhensif' à l'endroit de ses clients par exemple) ou que ce par quoi il est récompensé (la reconnaissance de son supérieur par exemple).

Notre recherche montre enfin la possibilité et l'intérêt d'une analyse qualitative d'échelles de mesure. Si de nombreux travaux s'inscrivant dans une perspective réaliste ont montré le manque de validité des échelles d'auto-évaluation de maladies chroniques (Baker, Stabile \& Deri, 2004 ; Johnston, Propper \& Shields, 2009), la méthodologie déployée met en lumière les hypothèses relatives à la 'nature' du répondant que portent de tels outils ; des hypothèses qui 
ne sont sans doute pas sans effet sur la manière dont l'individu se conçoit lui-même et sur les résultats de la recherche.

Ces résultats ouvrent un certain nombre de questionnements et pistes de recherche.

D'un point de vue théorique, ainsi, on peut se demander dans quelle mesure c'est la conception même de l'individu et les normes véhiculées notamment par les savoirs managériaux (les discours et les échelles d'auto-évaluation) qui ne sont pas elles-mêmes vecteurs de souffrance : l'individu souffre-t-il ainsi parce qu'il ne reçoit pas ce qu'il attend? et/ou parce qu'il n'a aucune prise sur ce qu'il peut attendre, parce que ce qu'on attend de lui s'impose ? ou encore parce qu'il est en quelque sorte pris en tenaille entre des discours valorisant la liberté et la réalisation de ses désirs, d'un côté, et des techniques de visibilité qui, avec d'autres dispositifs de management, supposent qu'il ne désire que ce que l'on attend de lui ? Souffrir, dans cette perspective, pourrait être analysé comme une forme de résistance à une exigence de non-action ou à la difficulté, voire l'impossibilité à satisfaire des prescriptions incompatibles.

Corrélativement, on peut se demander dans quelle mesure les traits ici rapportés participent de la définition que l'individu a de lui-même et de sa souffrance. Kalwak, Stupak et Bochaver (2011) ont ainsi montré un profond décalage entre les termes et expressions utilisés par les individus lorsqu'on leur demande de parler de leur souffrance physique et les termes utilisés dans les échelles d'auto-évaluation de cette même souffrance. Dans le prolongement de cette recherche, la comparaison des catégories et dimensions des échelles mises en évidence avec ce que l'individu dit de lui et de sa souffrance au travail dans le cadre d'entretien en profondeur par exemple, permettrait d'apprécier si les conceptions de la souffrance portées par les savoirs (théories, discours, échelles de mesure) participent ou non de sa subjectivité, i.e. du travail que l'individu opère sur lui-même. 
Plus largement, nos résultats invitent à analyser d'autres échelles couramment utilisées dans les recherches en gestion des ressources humaines (motivation, satisfaction, implication par exemple) pour apprécier dans quelle mesure elles participent ou non de la fabrication d'un même 'sujet'.

Au plan pratique enfin, les résultats de notre analyse invitent à sensibiliser les professionnels en charge de la prévention des risques psycho-sociaux (experts académiques, consultants, membres du CHSCT, représentants syndicaux et patronaux) à la conception du sujet que construisent les échelles de mesure de la souffrance utilisées. Certes, le déploiement d'enquêtes quantitatives témoigne de l'intérêt et de la 'prise en charge' par les directions, de la question de la souffrance au travail. Derrière leur apparente neutralité cependant, ces échelles véhiculent une certaine conception du sujet que les professionnels se devraient d'interroger: l'individu calculable, rationnel, mais également 'passif' à l'endroit de son organisation que construisent les échelles étudiées fait-il écho à la manière dont l'organisation voit l'individu au travail ? Quels sont les effets d'une telle conception?

S'il revient aux chercheurs de proposer d'autres instruments de mesure en vue de porter des conceptions différentes du sujet (un être plus actif, inscrit dans un collectif, une histoire, une culture, un individu acteur de la trajectoire de son organisation, préoccupé par la qualité de son travail par exemple), les résultats de cette recherche invitent à compléter les enquêtes quantitatives menées par un volet 'qualitatif'. Comme le montrent les travaux des psychodynamiciens du travail (e.g. Dejours, 2008), la conduite d'entretiens en profondeur ou de focus groups sont ainsi susceptibles de faire apparaitre des 'sujets' bien différents de ceux que portent les instruments de mesure étudiés (cf. Kalwak \& al., 2011). À moins, que ces instruments de mesure, aux côtés d'autres techniques managériales, ne soient parvenus à faire advenir ce sujet rationnel, calculateur et passif. Mais il s'agit, nous l'avons précédemment évoqué, d'une question méritant de bien plus amples investigations empiriques. 
Références

Baker, M., Stabile, M., \& Deri, C., (2004).What do self-reported objective measures of health measure?. Journal of Human Resources, 39(4), 1067-1093.

Bellinghausen, L., Collange, J., Botella, M., Emery, J.-L., \& Albert, E. (2009). Validation factorielle de l'échelle française de stress perçu en milieu professionnel. Santé Publique, 4(21), 365-373.

Bettschart, W., Plancherel, B., \& Bolognini, M. (1991). Validation du questionnaire de Goldberg (General Health Questionnaire, GHQ) dans un échantillon de population âgée de 20 ans. Psychologie médicale, 23(9), 1059-1064.

Bolognini, M., Bettschart, W., Zehndergubler, M., \& Rossier, L. (1989). The validity of the French version of the GHQ-28 and psydis in a community sample of 20 years old in Switzerland. European Archives of Psychiatry and Neurological Sciences, 238(3), 161168.

Clot, Y. (2010). Le Travail à Coeur. Paris : La Découverte.

Cohen, S., Kamarack, T., \& Mermerlstein, R. (1983). A global measure of perceived stress. Journal of Health and Social Behavior, 24(4), 385-396.

Cooper, C.L, Quick, J.C, \& Schabracq, M. J (2009). International Handbook of Work and Health Psychology ( $3^{\text {rd }}$ ed.). Chichester: Wiley Blackwell.

Daykin, N., \& Doyal, L. (1999). Health and Work. Critical Perspectives. Londres: Macmillan Press.

De Gaulejac, V. (2009). La Société Malade de la Gestion : Idéologie Gestionnaire, Pouvoir Managérial et Harcèlement Social. Paris : Points.

Dejours, C. (2008). Travail, Usure Mentale. Essai de Psychopathologie du Travail. Paris : Bayard.

Duncan, P. (2007). Critical Perspectives on Health. New York: Palgrave Macmilan.

Ezzamel, M., \& Willmott, H. (2010). Strategy and Strategizing: a Poststructuralist Perspective. In J. A. Baum \& J. Lampel (Eds.), The Globalization of Strategy Research, Advances in Strategic Management, vol. 27 (pp. 75-109). Bingley: Emerald Publishing.

Ezzamel, M., Willmott, H., \& Worthington, F. (2008). Manufacturing Shareholder Value: the Role of Accounting in Organizational Transformation. Accounting, Organizations and Society, 33(2-3), 107-140.

Foucault, M. (1969). L'Archéologie du Savoir. Paris: Gallimard.

Foucault, M. (1975). Surveiller et Punir. Naissance de la Prison. Paris: Gallimard.

Foucault, M. (1976a). Histoire de la Folie à l'Âge Classique. Paris: Gallimard.

Foucault, M. (1976b). Histoire de la Sexualité I. La Volonté de Savoir. Paris: Gallimard.

Foucault, M. (1978). La gouvernementalité, cours du Collège de France, 1977-1978, sécurité, territoire et population, 4ième leçon, 1er février 1978. Réédité in M. Foucault, 2001. Dits et écrits II, 1976-1988. Édition établie sous la direction de D. Defert \& F. Ewald, en coll. avec J. Lagrange (pp. 635-657). Paris : Gallimard. 
Foucault, M. (1983). L'écriture de soi. Corps écrit, 5, 3-23. Réédité in M. Foucault, 2001. Dits et écrits II, 1976-1988. Édition établie sous la direction de D. Defert \& F. Ewald, en coll. avec J. Lagrange (pp. 1234-1249). Paris : Gallimard.

Foucault, M. (1984). What is Enlightment? In H. Dreyfus \& P. Rabinow (Eds.), Michel Foucault: Beyond structuralism and hermeneutics (pp. 32-50). Chicago: The University of Chicago Press. Réédité in M. Foucault (2001). Dits et écrits II, 1976-1988 (pp. 13811397). Édition établie sous la direction de D. Defert \& F. Ewald, en coll. avec J. Lagrange Paris : Gallimard.

Foucault, M. (1988). Technologies of the self. In P. H. Hutton, H. Gutman \& L. H. Matrin (Eds.), Technologies of the self. A seminar with Michel Foucault (pp. 16-49). Anherst: The University of Massachusetts Press. Réédité et traduit in M. Foucault (2001). Dits et écrits II, 1976-1988. Édition établie sous la direction de D. Defert \& F. Ewald, en coll. avec J. Lagrange (pp. 1602-1632). Paris : Gallimard.

Foucault, M. (1991). Politics and the Study of Discourse. In G. Burchell, C. Gordon \& P. Miller (Eds.), The Foucault effect, Studies in Governmentality (pp. 53-72). Chicago: The University of Chicago Press.

Foucault, M. (2004). Naissance de la Biopolitique. Cours au Collège de France. 1978-1979. Paris: Gallimard/Seuil. Col. Hautes études.

Fox, N. J. (1999). Postmodern reflections: Deconstructing 'risk', 'health' and 'work'. In N. Daykin \& L. Doyal (Eds.), Health and Work. Critical Perspectives (pp. 198-219). Londres: Macmillan Press.

Girault, N. (1989). Burnout : émergence et stratégie d'adaptation, le cas de la médecine d'urgence, Thèse de Doctorat de Psychologie, Université René Descartes, Paris

Girault-Lidvan, N. (1996). Méthodes d'évaluation de l'épuisement professionnel. Limites et perspectives. Psychiatrie Française, 96(2), 30-39.

Glaser, B. \& Strauss, A. (1967). The Discovery of the Grounded Theory. Chicago: Aldine.

Goldberg, D. P, \& Hillier, V. F. (1979). A scaled version of the General Health Questionnaire. Psychological Medicine, 9(1), 139-145.

Golsorkhi, D., Huault, I., \& Leca, B. (2009). Les études critiques en management. Une perspective française. Québec: Les Presses de l'Université de Laval.

Hoskin, K. \& Macve, R. (1986). Accounting and the Examination: a Genealogy of Disciplinary Power. Accounting, Organizations and Society, 11(2), 105-136.

Huberman, M. \& Miles, M. (1984). Qualitative Data Analysis: A Source Book on New Methods. London: Sage.

Johnston, D. W., Propper, C., \& Shields, M. A. (2009). Comparing subjective and objective measures of health: Evidence from hypertension for the income/health gradient. Journal of Health Economics, 28(3), 540-552.

Kalwak, W., Stupak, R., \& Bochaver, A. (2011). Qualitative methods in pain research Adequacy of McGill pain questionnaire in the context of the qualia problem. International Society of Critical Health Psychology 7th Biennial Conference, 18-20th April, Adelaide Australia.

Karasek, R. (1979). Job demands, job decision latitude, and mental strain: implications for job design, Administrative Science Quaterly, 24(2), 285-308. 
Knights, D., \& McCabe, D. (2003). Governing through teamwork: Reconstituting subjectivity in a call center. Journal of Management Studies, 40(7), 1587-1619.

Knights, D., \& Morgan, G. (1991). Corporate Strategy, Organizations and the Subject: A Critique. Organization Studies, 12(2), 251-73.

Knights, D., \& Morgan, G. (1995). Strategic Management, Financial Services and Information Technology. Journal of Management Studies, 32(2), 191-214.

Knights, D., \& Willmott, H. (1989). Power and subjectivity at work: from degradation to subjugation in social relations. Sociology, 23(4), 535-58.

Lemyre, L., \& Tessier, R. (1988). Mesure du stress psychologique (MSP): Se sentir stressée, Canadian Journal of Behavioral Science, 20(3), 302-321

Lepine, J. P., Godchau, M., Brun, P., \& Lempériere, T. (1985). Evaluation de l'anxiété et de la dépression chez des patients hospitalisés dans un service de médecine interne, Annales Médico-Psychologiques, 143(2), 175-189.

Leplege A., Mesbah, M., Marquis, P. (1995). Preliminary analysis of the psychometric properties for the French version of an international questionnaire measuring the quality of life: the MOS SF 36 (version 1.1). Revue d'épidémiologie et de santé publique, 43(31), 371-379.

Lhuilier, D. (2010). Les «risques psychosociaux »: entre rémanence et méconnaissance, Nouvelle Revue de Psychosociologie, 2(10), 11-28.

Lilley, S. (2001). The Language of Strategy. In R. Westwood \& S. Linstead (Eds.), The Language of Organization (pp. 68-88). London: Sage Publications.

McKinlay, A., \& Starkey, K. (1998). Foucault, Management and Organization Theory. London: Sage.

Maslach, C., \& Jackson, S. E. (1981). The measurement of experienced burnout. Journal of Occupational Behavior, 2(2), 99-113

Maslach, C., Jackson, S. E., Leiter, M. P., Schaufeli, W. B., \& Schwab, R. L (2010). Maslach Burnout Inventory (MBI) Manual.

Miller, P., \& O'Leary, T. (1987). Accounting and the Construction of the Governable Person. Accounting. Organization and Society, 12(3), 235-265.

Nichols, T. (1999). Death and injury at work: A sociological perspective. In N. Daykin \& L. Doyal (Eds.), Health and Work. Critical Perspectives (pp. 86-106). Londres: Macmillan Press.

Niedhammer, I., Chastang, J.-F., Gendrey, L., David, S., \& Degioanni, S. (2006). Propriétés psychométriques de la version française des échelles de la demande psychologique, de la latitude décisionnelle et du soutien social du "Job Content Questionnaire" de Karasek : résultats de l'enquête nationale SUMER. Santé Publique, 18(3), 413-427

Oakes, L.S., Townley, B. and Cooper, D.J. (1998). Business Planning as Pedagogy: Language and Control in a Changing Institutional Field. Administrative Science Quarterly, 43(2), 257-292.

Payne, S. (1999). Paid and unpaid work in mental health : Towards a new perspective. In N. Daykin \& L. Doyal (Eds.), Health and Work. Critical Perspectives (pp. 35-53). Londres: Macmillan Press. 
Perret, V. (2009). Quand le changement devient soluble ou l'idéologie managériale du changement organisationnel. In D. Golsorkhi, I. Huault \& B. Leca (Eds.), Les études critiques en management. Une perspective française (pp. 209-231). Québec: Les Presses de l'Université de Laval.

Pezet, É. (2007). Introduction. In É. Pezet (Dir.), Management et conduite de soi: Enquête sur les ascèses de la performance (pp. 3-13). Paris: Vuibert.

Poisson J.F, (2008). Rapport sur la pénibilité au travail, http://www.assembleenationale.fr/13/rap-info/i0910-ti.asp.

Rose, N. (1999). Governing the Soul. The Shaping of the Private Self (2nd ed). London: Free Association Press.

Siegrist, J. (1996). Adverse health effects of high-effort/low-reward conditions. Journal of Occupational Health Psychology, 1, 27-41.

Shirom, A., \& Melamed, S. (2006). A comparison of the construct validity of two burnout measures in two groups of professionals. International Journal of Stress Management, 13, 176-200.

Steffy, B. D., \& Jones, J. W. (1988). Workplace stress and indicators of coronary disease risk. Academy of Management Journal, 31(3), 686-698.

Townley, B. (1993). Foucault, power/knowledge, and its relevance for human resource management. Academy of Management Review, 18(3), 528-545.

Townley, B. (1995). 'Know Thyself': Self-awareness, self-formation and managing. Organization, 2(2), 271-89.

Townley, B. (1998). Beyond Good and Evil: Depth and Division in the Management of Human Resources. In A. McKinlay \& K. Starkey (Eds.), Foucault, Management and Organization Theory (pp. 191-200). London: Sage.

Townley, B. (2002). Managing with modernity. Organization, 9(4), 549-573.

Verger P., Aulagnier, M., Schwoebel, V., \& Lang, T. (2005). Démarches épidémiologiques après une catastrophe. Anticiper les catastrophes: enjeux de santé publique, connaissances, outils et méthodes. - Réponses Environnement, Ministère de l'Ecologie et du Développement Durable. Paris : La Documentation Française.

Ware, J. E, \& Sherbourne, C. D, (1992). The MOS 36-item short-form health survey (SF-36) I. Conceptual framework and item selection. Medical Care, 30(6), 473-483.

Watterson, A. (1999). Why we still have old epidemics and endemics in occupational health: Policy and practice failures and some possible solutions. In N. Daykin \& L. Doyal (Eds.), Health and Work. Critical Perspectives (pp. 107-126). Londres: Macmillan Press.

Zigmond A. S., \& Snaith R. P. (1983). The Hospital Anxiety and Depression Scale. Acta Psychiatrica Scandinavica, 67(6), 361-370. 